\title{
SYSVENT: Prova de Conceito de um Protótipo para Ventilar Doentes em Cuidados Intensivos
}

\author{
SYSVENT: Proof of Concept Study of a Prototype to \\ Ventilate Critical Care Patients
}

\author{
Nuno CORTESÃO ${ }^{1}$, Marco FERNANDES ${ }^{2}$, Danielson PINA ${ }^{3}$, Felipe FERNANDES ${ }^{3}$, José Vale MACHADO³, \\ António H. CARNEIRO' 1
}

Acta Med Port 2021 Jan;34(1):12-19 - https://doi.org/10.20344/amp.14516

\section{RESUMO}

Introdução: A pandemia pelo novo coronavírus provocou rotura em hospitais de vários países por falta de recursos para ventilação invasiva. Assim, a Ordem dos Médicos convidou intensivistas que, em colaboração com a SYSADVANCE S.A., desenvolveram o SYSVENT OM1, um ventilador capaz de operar em modos controlados e assistidos (volume e pressão) e apto para tratar doentes em cuidados intensivos. Neste estudo fazemos a prova de conceito, comparando volume-corrente, pressão inspiratória e pressão positiva tele-expiratória programados, com os valores medidos pelo ventilador e por um equipamento de medição externo.

Material e Métodos: Montámos o ventilador em série com um pulmão artificial e um analisador de fluxos. Medimos o volume-corrente expiratório e a pressão inspiratória, em três níveis de compliance e seis patamares de volume-corrente. A pressão positiva tele-expiratória foi medida com incrementos de $2 \mathrm{cmH}_{2} \mathrm{O}$ ao longo de oito patamares. Para cada medição realizámos três leituras.

Resultados: Considerando cada uma das três variáveis isoladamente, a média da diferença máxima entre os valores programados e os valores medidos situa-se, para todas elas, dentro do que considerámos ser aceitável para um modelo protótipo (volume-corrente = $-28,1 \mathrm{~mL}$, pressão inspiratória $=0,8 \mathrm{cmH}_{2} \mathrm{O}$ e pressão positiva tele-expiratória $\left.=-1,1 \mathrm{cmH}_{2} \mathrm{O}\right)$. Essa diferença é maior quando avalia-da com o equipamento de medição externa comparativamente com o ventilador.

Discussão: Os resultados mostraram uma boa capacidade de monitorização e de precisão. Documentaram-se limitações técnicas relacionadas com o pulmão artificial e com o analisador de fluxos que não desvirtuam os resultados, mas limitam a sua amplitude.

Conclusão: Para os parâmetros testados, o ventilador apresenta boa performance de funcionamento, está de acordo com as premissas iniciais e tem potencial para uso clínico.

Palavras-chave: Cuidados Intensivos; Engenharia; Portugal; Respiração Artificial; Ventiladores Mecânicos

\section{ABSTRACT}

Introduction: The new coronavirus pandemic has led to scarcity of invasive ventilation resources in hospitals in several countries. In this context, the Portuguese Medical Association invited intensive care physicians who, in collaboration with SYSADVANCE S.A., developed SYSVENT OM1, a ventilator capable of operating in controlled and assisted modes (volume and pressure) and able to treat patients admitted to intensive care units. In this study we do the proof of concept comparing programmed tidal volume, inspiratory pressure and positive end-expiratory pressure with those measured by the ventilator and an external measuring equipment.

Material and Methods: We set up the ventilator in tandem with an artificial lung and a flow analyzer. We measured expiratory tidal volume, and inspiratory pressure against three levels of compliance, each with six steps of tidal volume. Positive end-expiratory pressure was measured at $2 \mathrm{cmH}_{2} \mathrm{O}$ incremental along eight steps. For each measurement, we performed three readings.

Results: Considering each of the three single variables, the mean value of the highest difference between programmed values and measured values is, for all of them, within what we considered to be acceptable for a prototype model (tidal volume $=-28.1 \mathrm{~mL}$, inspiratory pressure $=0.8 \mathrm{cmH}_{2} \mathrm{O}$ and positive end-expiratory pressure $=-1.1 \mathrm{cmH}_{2} \mathrm{O}$ ). This difference is greater when evaluated with the external measuring equipment in comparison with the ventilator.

Discussion: The results showed a good monitoring and accuracy performance. Technical limitations related with the artificial lung and the flow analyzer have been documented, which do not compromise the results, but limit their amplitude.

Conclusion: For tested parameters, the ventilator has a good operating performance, is in accordance with the initial premises and has potential for clinical use.

Keywords: Critical Care; Engineering; Portugal; Respiration, Artificial; Ventilators, Mechanical

\section{INTRODUÇÃO}

A atual pandemia pelo SARS-CoV-2 (COVID-19) surpreendeu muitos países, quer pela exigência súbita e massiva de recursos diferenciados, quer pelas intervenções para controlo da disseminação da infeção. A disfunção respiratória tem sido a forma mais prevalente de manifestação grave da doença e é a principal causa quer de admissão em unidades de cuidados intensivos (UCI), quer de mortalidade. A insuficiência respiratória é predominantemen- te hipoxémica, grave, evoluindo umas vezes sob a forma de acute respiratory distress syndrome (ARDS) clássico, outras vezes sob a forma de um envolvimento pulmonar com compliance elevada e hipoxemia grave associadas a mecanismo de shunt intrapulmonar. A utilização de equipamentos de oxigenação e de ventilação não-invasiva para o suporte da insuficiência respiratória não tem sido consensual pelo potencial risco de contágio associado

1. Unidade de Cuidados Intensivos. Departamento de Medicina. Hospital da Luz - Arrábida. Vila Nova de Gaia. Portugal.

2. Serviço de Medicina Intensiva Polivalente. Centro Hospitalar de Entre Douro e Vouga. Santa Maria da Feira. Portugal.

3. SYSADVANCE, S.A.. Póvoa de Varzim. Portugal.

$\triangle$ Autor correspondente: Nuno Cortesão. ncortesao@gmail.com

Recebido: 08 de julho de 2020 - Aceite: 19 de outubro de 2020 | Copyright ( $)$ Ordem dos Médicos 2021 
(aerossolização). Como consequência, os países atingidos têm assumido a tarefa de dotar os seus sistemas de saúde com um número adequado de equipamentos destinados a ventilação invasiva por forma a responder às necessidades esperadas. Este cenário tem colocado uma enorme pressão na comercialização destes produtos. A experiência demonstrou que em muitos países não foi possível suprir, em tempo oportuno, a necessidade acrescida de recursos para ventilação invasiva. Por outro lado, os países que não dispunham de capacidade de produção dos seus próprios equipamentos ficaram dependentes de exigências de mercado liberalizado, que nem sempre permitiu responder às necessidades como seria desejável e necessário.

Neste contexto, a empresa SYSADVANCE, S.A., em parceria com intensivistas convidados pela Ordem dos Médicos (OM) e com a Associação Empresarial de Portugal (AEP), desenvolveu um ventilador para ventilação invasiva em adultos - SYSVENT OM1 (SYSVENT). A SYSADVANCE, SA, iniciou a sua atividade em 2002, como spin-off de um laboratório universitário de Investigação e Desenvolvimento (I\&D), e dedica-se a desenvolver e comercializar tecnologia de manipulação, tratamento e fabrico de gases e purificadores de gases, bem como soluções integradas para ar comprimido. O sistema de gestão da qualidade (QMS) da SYSADVANCE é certificado de acordo com a norma ISO 9001 para toda a gama de produtos industriais e do setor energético. O SYSADVANCE QMS também é certificado de acordo com a norma ISO 13485 para dispositivos médicos geradores de oxigénio médico e sistemas de geração de oxigénio, sistemas médicos de ar e vácuo. Estes dispositivos médicos são certificados de acordo com a Diretiva 93/42/CEE de dispositivos médicos.

O SYSVENT foi desenvolvido com base em pressupostos e requisitos pré-definidos por médicos intensivistas indigitados pela OM para este efeito. Para este ventilador de concepção portuguesa, foram definidos como premissas a construção de um produto simples, fiável, robusto, financeiramente viável e capaz de garantir a sua aplicação clínica cumprindo os standards vigentes. ${ }^{1-3}$

O objetivo deste estudo foi desenvolver uma prova de conceito, comparando o volume-corrente $\left(\mathrm{V}_{\mathrm{T}}\right)$, a pressão inspiratória $\left(P_{\text {insp }}\right)$ e a pressão positiva tele-expiratória (PEEP) programados no ventilador SYSVENT (os dois primeiros programados nos modos volume-controlado e pressão-controlada, respetivamente), em três níveis pré-definidos de compliance (10, 20 e 50 mlL/ $\mathrm{cmH}_{2} \mathrm{O}$ ), com aqueles medidos pelo próprio ventilador (monitorização) e por um equipamento de medição externo.

\section{MATERIAL E MÉTODOS \\ Equipamentos \\ SYSVENT OM1}

O protótipo do SYSVENT destina-se a ventilação invasiva de adultos, é operado por $\mathrm{O}_{2}$ comprimido, tem um peso aproximado de $22 \mathrm{~kg}$ e incorpora uma bateria interna (UPS) com autonomia para uma hora.

A interface do operador com o ventilador faz-se por in- termédio de um monitor de programação tátil, que serve simultaneamente para programação e monitorização ciclo a ciclo. A tecnologia utilizada permite também que a programação e a monitorização possam ser feitas à distância, facto que reduz a necessidade de contacto direto com o ventilador ou com o ambiente em que o doente se encontra. O ventilador incorpora os modos pressão-controlada (PC) e pressão assistida-controlada (PA/C), modo volume-controlado (VC) e volume assistido-controlado (VA/C) e modo assistido (A). As amplitudes e unidades de medida para as variáveis programáveis são as que se detalham na Tabela 1.

A monitorização expressa-se sob a forma de valores absolutos de fração inspirada de oxigénio $\left(\mathrm{FiO}_{2}\right)$, frequência respiratória (FR), PEEP, $\mathrm{P}_{\text {insp }}, \mathrm{V}_{\mathrm{Te}}$ (volume-corrente expiratório), VM, ratio inspiração:expiração (ratio I:E) e sob a forma gráfica para $F, V_{T}$ e $P$, em função do tempo. Estão também contempladas pausa inspiratória ( Pausa $_{\text {insp }}$ ) e expiratória $\left(\right.$ Pausa $\left._{\text {exp }}\right)$ para medição, respetivamente, da pressão de planalto tele-inspiratório $\left(P_{\text {plateau }}\right)$ e de PEEP. Estes procedimentos especiais permitem, por conseguinte, o cálculo automático da compliance estática $\left(\mathrm{C}_{\mathrm{st}}\right)$ e da resistência das vias aéreas $(R)$. Os alarmes estão hierarquizados em dois níveis de alerta de acordo com o risco para o doente. Os atributos distintivos para cada alarme são um sinal luminoso e sonoro. O alarme standard é atribuído a $\mathrm{FR}, \mathrm{V}_{\mathrm{T}}, \mathrm{P}_{\text {insp }}$ e VM. O alarme crítico é atribuído à desconexão, apneia (com modo de ventilação de backup) e fuga. Ao contrário dos restantes alarmes, o alarme de desconexão e fuga não são programáveis. Os alarmes $\mathrm{V}_{\mathrm{T}}$ e $\mathrm{P}_{\text {insp }}$ são ativos no sentido em que os limites de alarme definidos para estes parâmetros se sobrepõem àqueles que venham a ser programados.

\section{Pulmão artificial}

Utilizámos um pulmão artificial (lung ventilator performance analyzer - Medishield) que permite variar $\mathrm{R}\left(0,5,20,50,200 \mathrm{cmH}_{2} \mathrm{O} / \mathrm{L} / \mathrm{s}\right)$, a fuga (variável, mas

Tabela 1 - Unidades de medidas e amplitudes de operação de cada parâmetro de programação

\begin{tabular}{lcc}
\hline \multicolumn{1}{c}{ Parâmetro } & Unidade & Amplitude \\
\hline $\mathrm{FiO}_{2}$ & $\%$ & {$[0,21 ; 1]$} \\
$\mathrm{F}$ & $\mathrm{L} / \mathrm{min}$ & {$[2 ; 120]$} \\
$\mathrm{FR}$ & $\mathrm{cpm}$ & {$[0,5 ; 40]$} \\
$\mathrm{PEEP}$ & $\mathrm{cmH}_{2} \mathrm{O}$ & {$[1 ; 20]$} \\
$\mathrm{P}_{\text {insp }}$ & $\mathrm{cmH}_{2} \mathrm{O}$ & {$[1 ; 80]$} \\
$\mathrm{PS}$ & $\mathrm{cmH}_{2} \mathrm{O}$ & {$[0 ; 80]$} \\
$\mathrm{RT}$ & $\mathrm{S}$ & {$[0,1 ; 2]$} \\
Term & $\%$ & {$[5 ; 30]$} \\
$\mathrm{T}_{\mathrm{i}}$ & $\mathrm{s}$ & {$[0,3 ; 10]$} \\
Trigger & $\mathrm{cmH}_{2} \mathrm{O}$ & {$[1 ; 10]$} \\
$\mathrm{V}_{\mathrm{T}}$ & $\mathrm{mL}$ & {$[0,1 ; 1.5]$} \\
\hline
\end{tabular}

$\mathrm{FiO}_{2}$ : fração inspirada de oxigénio; $\mathrm{F}$ : fluxo inspiratório; FR: frequência respiratória; PEEP: positive end-expiratory pressure; $\mathrm{P}_{\text {insp }}$ : pressão inspiratória; $P S$ : pressão de suporte; RT: rise time; Term $_{\text {insp }}$ : ciclagem; $\mathrm{T}_{\mathrm{i}}$ : tempo inspiratório; $\mathrm{V}_{\mathrm{T}}$ : volume-corrente 


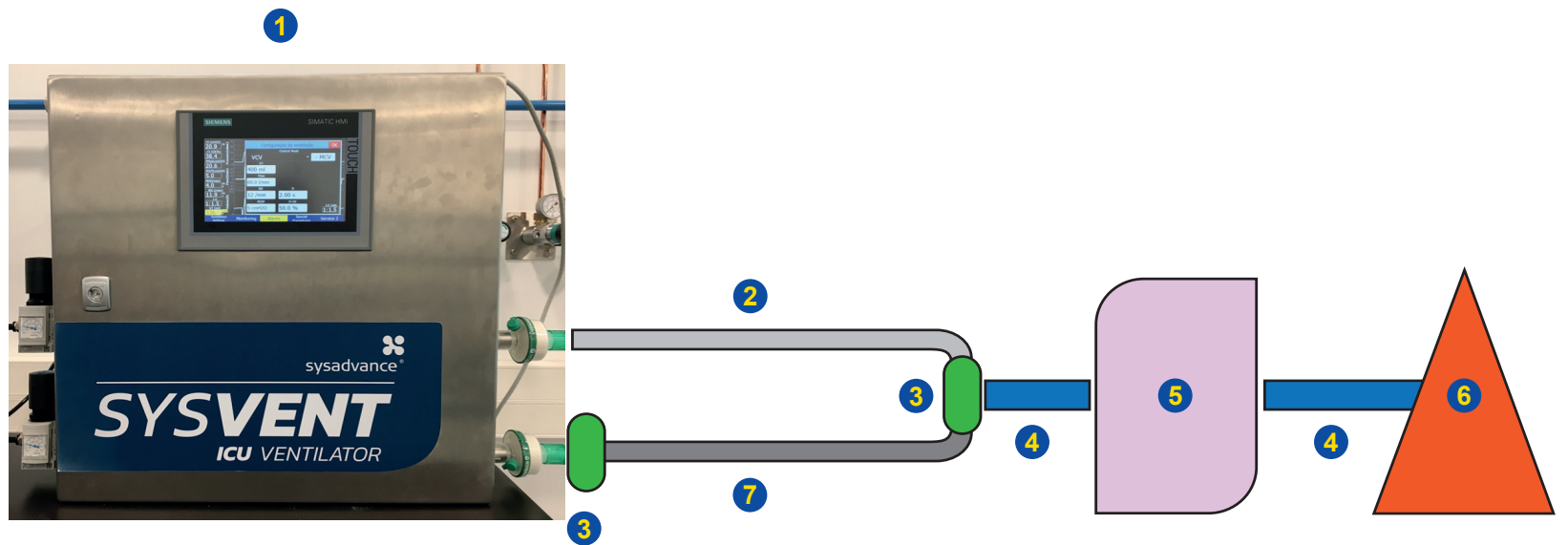

Figura 1 - Esquema da montagem dos equipamentos para os testes de bancada. 1. Ventilador SYSVENT; 2. Ramo inspiratório; 3. Filtro antibacteriano + HME (Inter-ThermFilter/HME da Intersurgical); 4. Tubo; 5. Bio-Tek; 6. Pulmão artificial; 7. Ramo expiratório

adimensional) e a compliance $(\mathrm{C})$ em três níveis distintos $\left(10,20\right.$ e $\left.50 \mathrm{~mL} / \mathrm{cmH}_{2} \mathrm{O}\right)$.

\section{Analisador de fluxos de gases}

Como instrumento de medição externa, usámos o Bio-Tek VT Plus Gas Flow Analizer - Fluke Biomedical (Bio-Tek), que permite a medição em tempo real e síncrona de diferentes parâmetros volumétricos e pressurimétricos.

\section{Montagem do circuito dos equipamentos}

A montagem dos equipamentos em causa está exemplificada na Fig. 1.

\section{Medições}

Para a medição de $\mathrm{V}_{\mathrm{T}}$ e $\mathrm{P}_{\text {insp }}$, programámos o ventilador com FR $=12 \mathrm{cpm}, \mathrm{PEEP}=5 \mathrm{cmH}_{2} \mathrm{O}$, fluxo $(\mathrm{F})=60 \mathrm{~L} / \mathrm{min}$ (para $\mathrm{PC}$ ) e $40 \mathrm{~L} / \mathrm{min}$ (para $\mathrm{VC}$ ), tempo inspiratório $\left(\mathrm{T}_{\mathrm{i}}\right)=1 \mathrm{~s}$ (para $\mathrm{PC}$ ) e 1,2s (para VC), $\mathrm{R}=0 \mathrm{cmH}_{2} \mathrm{O} / \mathrm{L} / \mathrm{s}$ (para além da imposta pela montagem), fuga $=0 \mathrm{~L} / \mathrm{min}$. Para ambos os modos ventilatórios e para um peso corporal teórico de $70 \mathrm{~kg}$, definimos um incremento de $1 \mathrm{~mL} / \mathrm{kg}$, entre um patamar mínimo de $5 \mathrm{~mL} / \mathrm{kg}$ e um patamar máximo de $10 \mathrm{~mL} /$ $\mathrm{kg}$, incluindo, portanto, o âmbito de $\mathrm{V}_{\mathrm{T}}$ recomendado para a utilização clínica com uma estratégia protetora do pulmão (6 a $8 \mathrm{~mL} / \mathrm{kg}$ de peso ideal). Foram testados os volumes $350 \mathrm{~mL}, 420 \mathrm{~mL}, 490 \mathrm{~mL}, 560 \mathrm{~mL}, 630 \mathrm{~mL}$ e $700 \mathrm{~mL}$. Para cada patamar de $V_{T}$, fizemos três medições consecutivas, com leitura de $\mathrm{V}_{\mathrm{T}}$ expirado $\left(\mathrm{V}_{\mathrm{Te}}\right)$ no final de uma pausa inspiratória de quatro segundos. No caso do modo $\mathrm{VC}, \mathrm{V}_{\mathrm{T}}$ foi diretamente programado no ventilador. No caso do modo $\mathrm{PC}$, determinámos a pressão que mais se aproximou de $\mathrm{V}_{\mathrm{T}}$ previsto para cada patamar, simulando, assim, a prática clínica habitual. Estas medições foram efetuadas para três níveis de $\mathrm{C}$ pré-definidos $\left(10,20\right.$ e $\left.50 \mathrm{~mL} / \mathrm{cmH}_{2} \mathrm{O}\right)$ no pulmão artificial. A PEEP foi avaliada com incrementos de $2 \mathrm{cmH}_{2} \mathrm{O}$, entre 0 e $16 \mathrm{cmH}_{2} \mathrm{O}$, com base numa parametrização de $V_{T}=400 \mathrm{~mL}, F R=12 \mathrm{cpm}, \mathrm{F}=40 \mathrm{~L} / \mathrm{min}, \mathrm{T}_{\mathrm{i}}=1 \mathrm{~s}$, $\mathrm{C}=20 \mathrm{~mL} / \mathrm{cmH}_{2} \mathrm{O}, \mathrm{R}=0 \mathrm{cmH}_{2} \mathrm{O} / \mathrm{L} / \mathrm{s}$ (para além da imposta pela montagem) e fuga $=0 \mathrm{~L} / \mathrm{min}$. Todas as medições foram feitas em condições ambient temperature, pressure, and saturated with water vapour (ATPS).

Os equipamentos de simulação e medida utilizados apresentam duas limitações técnicas que importa clarificar:

1. Verificámos o bom funcionamento do modo assistido, com resposta pressurimétrica adequada, sempre que foi simulado um apelo inspiratório. Esta foi, porém, uma análise subjetiva, não mensurável, atendendo a que o equipamento de medição de que dispúnhamos na altura não permitia titular a pressão do apelo inspiratório simulado para o limiar de sensibilidade do trigger definido. Assim, os autores decidiram anunciar, tão somente, que este modo ventilatório está disponível nesta versão protótipo, sem descrever o seu detalhe nem proceder a medições;

2. Para um nível de compliance pulmonar de $50 \mathrm{~mL} /$ $\mathrm{cmH}_{2} \mathrm{O}$, e para $\mathrm{V}_{\mathrm{T}}$ mais elevados $(8 \mathrm{~mL} / \mathrm{kg}, 9 \mathrm{~mL} / \mathrm{kg}$ e 10 $\mathrm{mL} / \mathrm{kg}$, correspondendo a 560, 630 e $700 \mathrm{~mL}$ ), o pulmão artificial, por defeito técnico, não permitia a insuflação da totalidade do volume programado.

A aquisição de um novo pulmão artificial e instrumento de medição, permitirá, no curto-prazo, ultrapassar estas limitações e completar os testes de bancada, solucionando as limitações acima descritas.

\section{Estatística}

Foi efetuada uma análise descritiva das diferenças entre os valores medidos e os parâmetros programados para $V_{T}, P_{\text {insp }}$ e PEEP. Para cada uma dessas variáveis foi aplicado o teste não-paramétrico de Kolmogorov-Smirnov, que não encontrou argumentos para a rejeição duma distribuição normal. Assim, a média foi usada como medida de tendência central e o desvio-padrão como medida de dispersão. Para o efeito, utilizámos o software de estatística IBM SPSS Statistics Versão 26.

\section{RESULTADOS}

\section{Volume-controlado}

A média global da diferença entre $V_{T}$ programado e medido foi de $-28,1 \mathrm{~mL}$ para o Bio-Tek e $6,2 \mathrm{~mL}$ para o 
Tabela 2 - Caracterização da diferença entre $\mathrm{V}_{\mathrm{T}}$ programado e $\mathrm{V}_{\mathrm{Te}}$ medido

\begin{tabular}{|c|c|c|c|c|c|c|c|}
\hline & & \multicolumn{6}{|c|}{$\begin{array}{c}\text { C programada } \\
\left(\mathrm{mL} / \mathrm{cmH}_{2} \mathrm{O}\right)\end{array}$} \\
\hline & & \multicolumn{2}{|c|}{10} & \multicolumn{2}{|c|}{20} & \multicolumn{2}{|c|}{50} \\
\hline & & SYSVENT & Bio-TEK & SYSVENT & Bio-TEK & SYSVENT & Bio-TEK \\
\hline \multirow{7}{*}{ 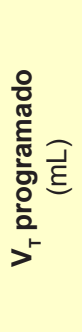 } & 350 & $9,0(3,0)$ & $-19,7(2,5)$ & $-4,7(7,5)$ & $-14,7(8,6)$ & $5,0(6,1)$ & $-8,7(1,2)$ \\
\hline & 420 & $7,3(5,5)$ & $-29,3(3,2)$ & $3,0(10,1)$ & $-18,3(3,8)$ & $7.3(1,2)$ & $-11,3(3,2)$ \\
\hline & 490 & $14,7(2,1)$ & $-2,7(52,6)$ & $8,0(2,0)$ & $-24,0(3,0)$ & $6,3(4,0)$ & $-16,0(1,7)$ \\
\hline & 560 & $8,3(2,5)$ & $-46,3(7,6)$ & $2,3(3,8)$ & $-34,3(2,5)$ & $\mathrm{N} / \mathrm{A}$ & $\mathrm{N} / \mathrm{A}$ \\
\hline & 630 & $10,3(10,7)$ & $-48,7(0,6)$ & $7,3(1,2)$ & $-37,3(1,5)$ & $\mathrm{N} / \mathrm{A}$ & $\mathrm{N} / \mathrm{A}$ \\
\hline & 700 & $3,3(2,1)$ & $-61,0(4,4)$ & $5,7(10,3)$ & $-48,7(7,5)$ & $\mathrm{N} / \mathrm{A}$ & $\mathrm{N} / \mathrm{A}$ \\
\hline & Global & $8,8(5,7)$ & $-34,6(27,3)$ & $3,6(7,3)$ & $-29,6(12,9)$ & $6,2(3,8)$ & $-14,9(3,7)$ \\
\hline
\end{tabular}

A tabela mostra a média e desvio-padrão da diferença entre $V_{T}$ programado e $V_{T e}$ medido pelo SYSVENT e pelo Bio-Tek, expresso em mililitros, para cada patamar de $V_{T}$ programado e para cada nível de C. A última linha agrega todos os dados numa medida global, que espelha as medições parcelares.

SYSVENT. Em termos percentuais, a média dessas diferenças corresponde a $-5,3 \%$ para o Bio-Tek e 1,3\% para o SYSVENT. Quando se comparam as diferenças das medições entre o SYSVENT e o Bio-Tek (Tabela 2), para cada patamar e para cada nível de $\mathrm{C}$, verificámos que as primeiras estão mais próximas do programado (média do maior desvio $=14,7 \mathrm{~mL}$ ) e são de sinal positivo. Com o Bio-Tek, as medições são, por regra, inferiores às programadas e divergem delas de forma mais relevante (média do maior desvio $=-61 \mathrm{~mL}$ ). Acresce ainda que, ao contrário do que ocorre com o SYSVENT (que mantém uma diferença estável ao longo dos diferentes patamares de $\mathrm{V}_{\mathrm{T}}$ e de níveis de $\mathrm{C}$, com médias que variam de $-4,7 \mathrm{~mL}$ a $14,7 \mathrm{~mL}$ ), com o Bio-Tek o desvio do programado aumenta com o incremento de $\mathrm{V}_{\mathrm{T}}$ programado em todos os níveis de $\mathrm{C}$, passando de $-2,0 \mathrm{~mL}$ a $-61,0 \mathrm{~mL}$ (Tabela 2).

A expressão gráfica dessas diferenças (Fig. 2) vem mostrar que, com o Bio-Tek, e contrariamente ao que ocorre com o SYSVENT, a divergência das medições aumenta ao longo dos patamares de $\mathrm{V}_{\mathrm{T}}$ programados e em todos os

\section{C programada $\left(\mathrm{mL} / \mathrm{cmH}_{2} \mathrm{O}\right)$}

10

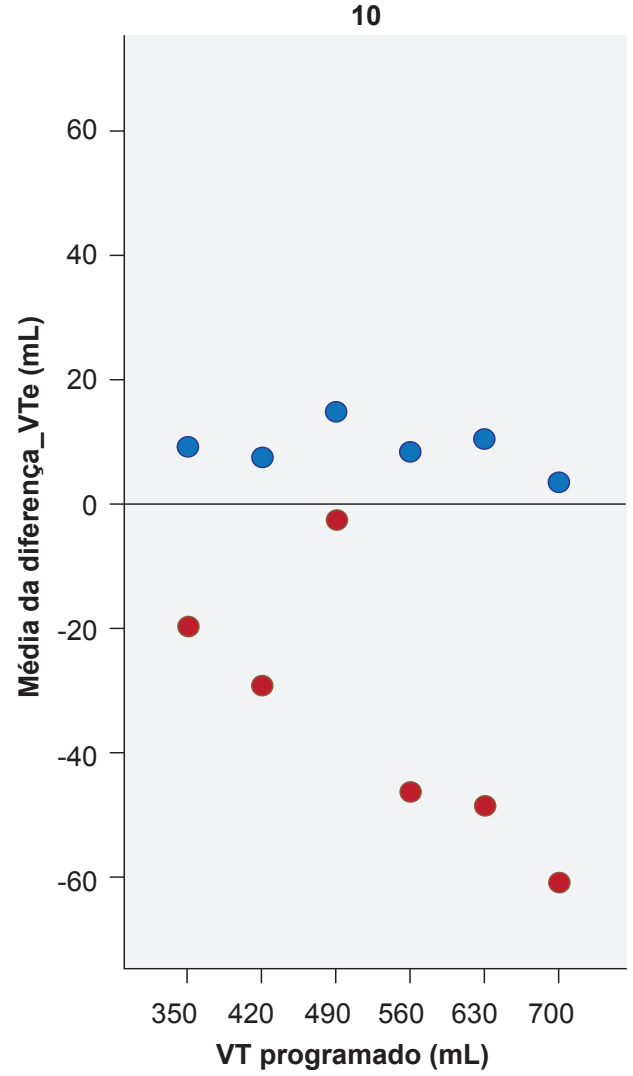

20

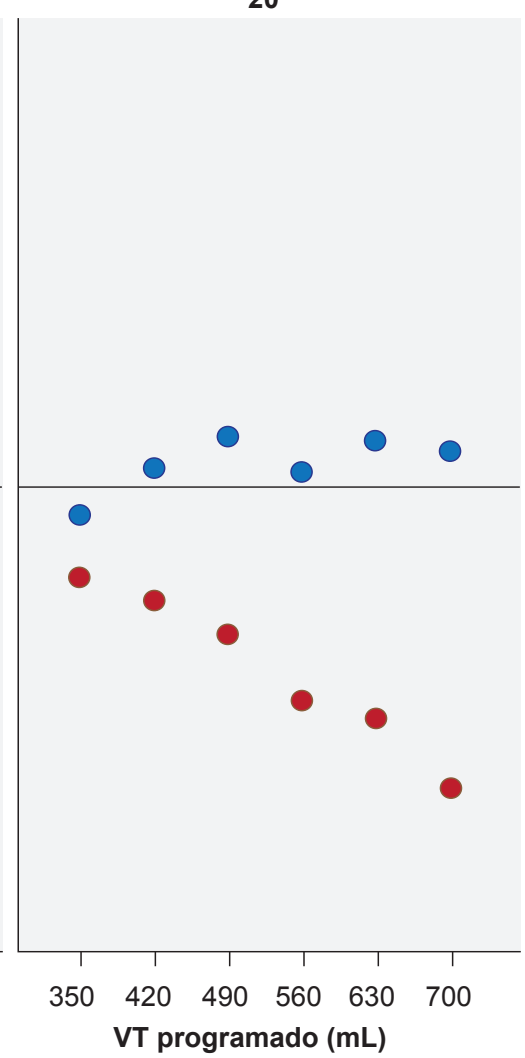

50

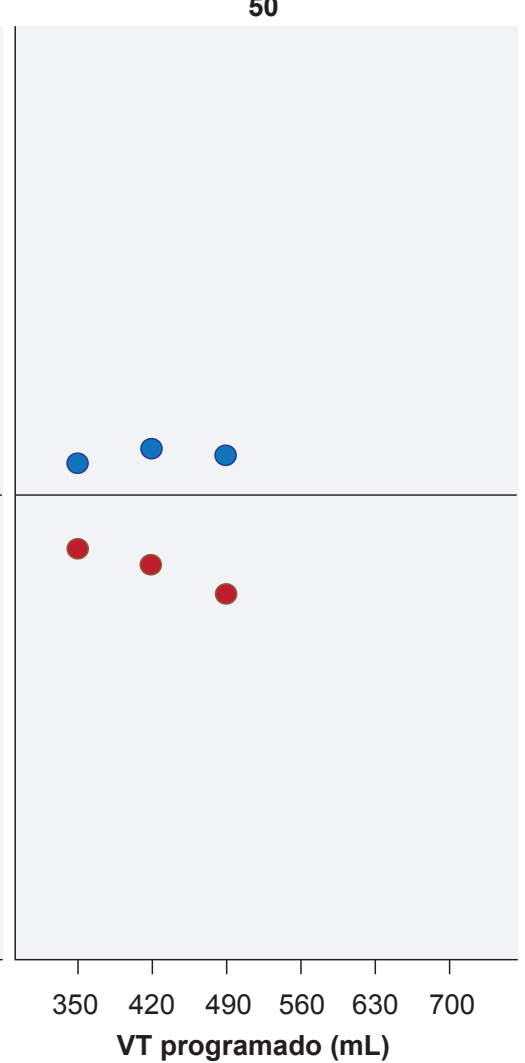

SYSVENT

Bio-TEK

Figura 2 - Distribuição das médias das diferenças, expressas em mL, entre $\mathrm{V}_{\mathrm{Te}}$ medido no SYSVENT e no Bio-Tek para cada patamar de $\mathrm{V}_{\mathrm{T}}$ programado e para cada nível de $\mathrm{C}$ 
níveis de $\mathrm{C}$ considerados, particularmente para os níveis de $\mathrm{C}=10 \mathrm{~mL} / \mathrm{cmH}_{2} \mathrm{O}$ e $\mathrm{C}=20 \mathrm{~mL} / \mathrm{cmH}_{2} \mathrm{O}$. Para o nível de $\mathrm{C}=50 \mathrm{~mL} / \mathrm{cmH}_{2} \mathrm{O}$, essa divergência parece ser menor, embora a interpretação esteja limitada pelo facto de nem todos os patamares de VT terem sido testados (vide supra).

\section{Pressão-controlada}

A média global da diferença foi de $0,8 \mathrm{cmH}_{2} \mathrm{O}$, quer para o SYSVENT, quer para o Bio-Tek (Tabela 3). A distribuição dessas diferenças em função das $P_{\text {insp }}$ programadas e estratificada pela C (Fig. 3) mostra que há sobreposição das

Tabela 3 - Caracterização da diferença entre $\mathrm{P}_{\text {insp }}$ programada e medida

\begin{tabular}{|c|c|c|c|}
\hline & & \multicolumn{2}{|c|}{$\begin{array}{c}\text { Diferença_Pinsp } \\
\left(\mathrm{cmH}_{2} \mathrm{O}\right)\end{array}$} \\
\hline & & SYSVENT & Bio-Tek \\
\hline & & Média (SD) & Média (SD) \\
\hline \multirow{4}{*}{ 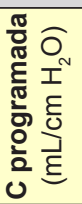 } & 10 & $1,5(1,1)$ & $1,5(1,0)$ \\
\hline & 20 & $0,3(0,2)$ & $0,3(0,1)$ \\
\hline & 50 & $0,6(0)$ & $0,3(0)$ \\
\hline & Global & $0,8(0,9)$ & $0,8(0,9)$ \\
\hline
\end{tabular}

A tabela mostra a média e desvio-padrão da diferença entre $P_{\text {insp }}$ programada e a medida pelo SYSVENT e pelo Bio-Tek, expressa em $\mathrm{cmH}_{2} \mathrm{O}$, global e para cada nível de C definida. medições efectuadas pelo SYSVENT e pelo Bio-Tek, e que a magnitude do desvio em relação aos valores programados não é relevante sob o ponto de vista clínico. Para um nível de $\mathrm{C}=10 \mathrm{~mL} / \mathrm{cmH}_{2} \mathrm{O}$, parece haver uma tendência de aumento das diferenças para pressões mais elevadas, com um valor máximo de cerca de $4 \mathrm{cmH}_{2} \mathrm{O}$. Todavia, esse aumento continua a não ter relevância clínica até porque ocorre para um nível de $\mathrm{P}_{\text {insp }}$ programada que não é utilizada, nem recomendada, para a prática clínica.

\section{PEEP}

A média da diferença entre PEEP programada e medida foi de $-1,1 \mathrm{cmH}_{2} 0$ e de $-0,6 \mathrm{cmH}_{2} \mathrm{O}$ para o Bio-Tek e para o SYSVENT, respetivamente (Tabela 4). A média destas diferenças, quando distribuídas ao longo de PEEP programada (Fig. 4), continua a ser de valor inferior para o Bio-Tek quando comparado com o SYSVENT. Por outro lado, com o incremento de PEEP programada, a média das diferenças aumenta, quer para o SYSVENT, quer para o Bio-Tek embora, sublinha-se, sem significado clínico.

\section{DISCUSSÃO}

Genericamente, os resultados dos testes de bancada ao ventilador SYSVENT OM1 mostram uma boa capacidade de monitorização (os valores medidos pelo próprio
10

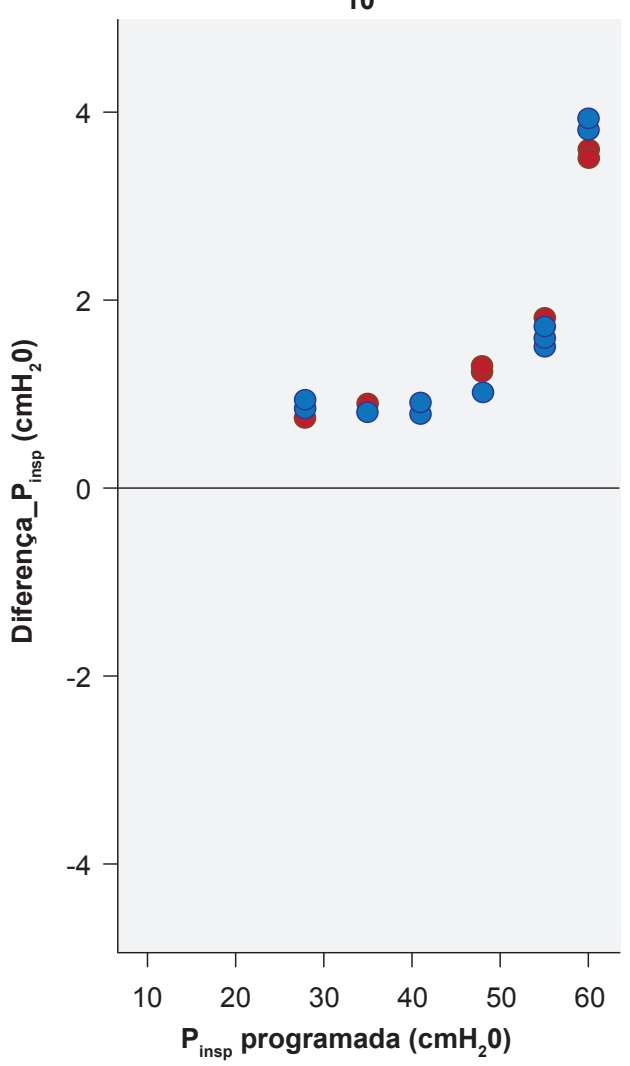

C programada $\left(\mathrm{mL}_{\mathrm{cmH}} \mathrm{O}\right)$

20

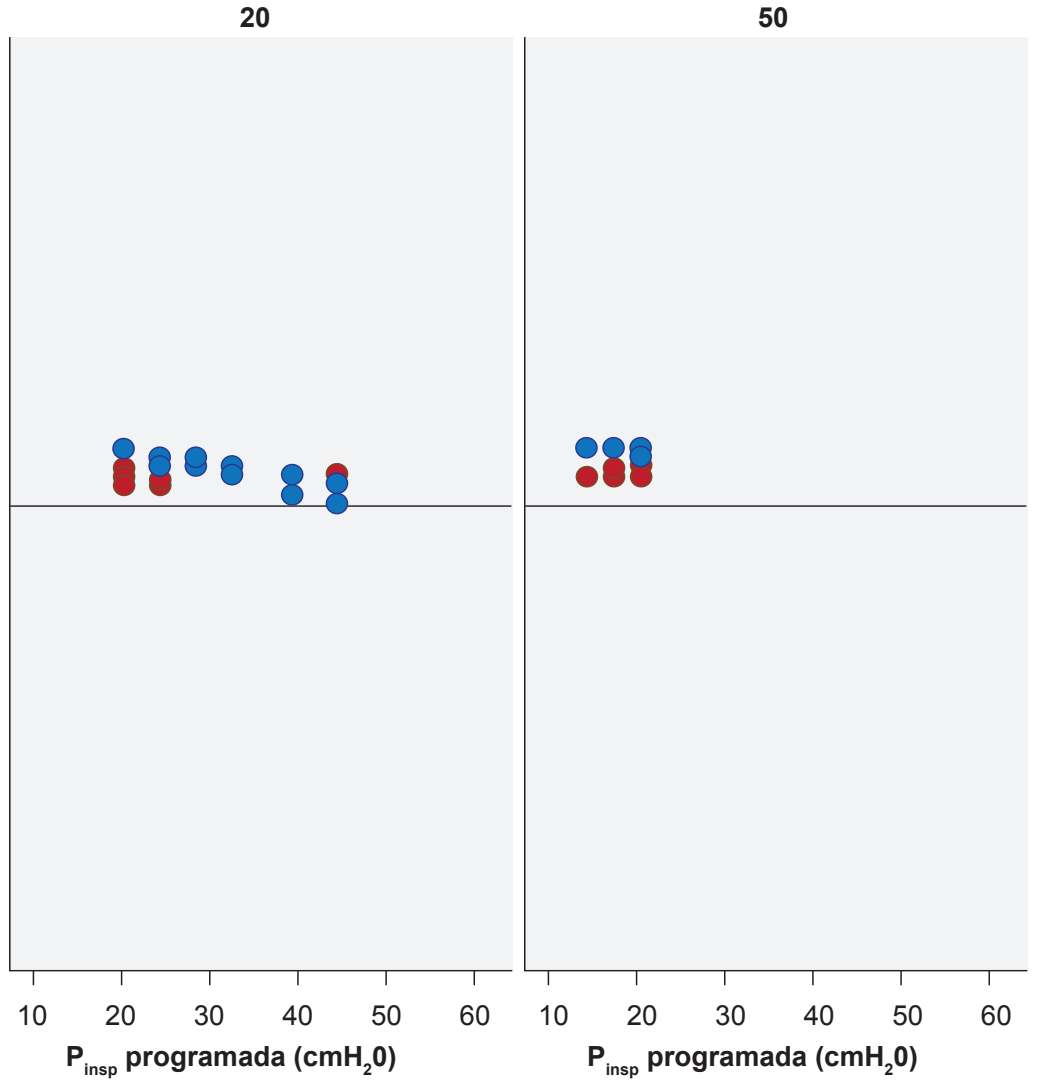

SYSVENT

Bio-TEK

Figura 3 - Distribuição das diferenças, expressas em $\mathrm{cm} \mathrm{H}_{2} \mathrm{O}$, entre $\mathrm{P}_{\text {insp }}$ medida no SYSVENT e no Bio-Tek para cada $\mathrm{P}_{\text {insp }}$ programado e para cada nível de $\mathrm{C}$ 
Tabela 4 - Caracterização da diferença entre PEEP programada e medida

\begin{tabular}{|c|c|c|c|}
\hline & & \multicolumn{2}{|c|}{$\begin{array}{c}\text { Diferença_PEEP } \\
\left(\mathrm{cmH}_{2} \mathrm{O}\right)\end{array}$} \\
\hline & & SYSVENT & Bio-Tek \\
\hline & & Média (SD) & Média (SD) \\
\hline \multirow{10}{*}{ 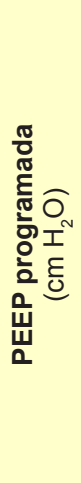 } & 0 & $0,5(0)$ & $0(0)$ \\
\hline & 2 & $0,1(0)$ & $-0,6(0)$ \\
\hline & 4 & $-0,6(0)$ & $-1,3(0,2)$ \\
\hline & 6 & $-0,8(0,1)$ & $-1,3(0,2)$ \\
\hline & 8 & $-0,9(0,1)$ & $-1,5(0,3)$ \\
\hline & 10 & $-0,8(0,1)$ & $-1,2(0,2)$ \\
\hline & 12 & $-1,0(0,1)$ & $-1,3(0,2)$ \\
\hline & 14 & $-1,1(0,1)$ & $-1,4(0,1)$ \\
\hline & 16 & $-1,1(0,1)$ & $-1,4(0,2)$ \\
\hline & Global & $-0,6(0,5)$ & $-1,1(0,5)$ \\
\hline
\end{tabular}

A tabela mostra a média e desvio-padrão das diferenças entre PEEP programada e a medida pelo SYSVENT e pelo Bio-Tek, expressa em $\mathrm{cmH}_{2} \mathrm{O}$, global e para cada nivel de PEEP definida. equipamento são próximos dos programados) e de precisão (as diferenças entre o programado e o medido pelo Bio-Tek são clinicamente aceitáveis).

No caso de $\mathrm{V}_{\mathrm{T}}$, a literatura aponta uma diferença entre $\mathrm{V}_{\mathrm{Te}}$ medido e programado que varia de $-5 \%$ a $20 \%$. $^{4-}$ 7 No nosso estudo, a média global desta diferença variou de $-5,3 \%$ a $1,2 \%$, consoante se trate de $\mathrm{V}_{\mathrm{Te}}$ medido pelo SYSVENT ou de $\mathrm{V}_{\mathrm{Te}}$ medido pelo Bio-Tek. Estes resultados comparam com o descrito na literatura, corroborando a boa precisão do ventilador. As medições feitas pelo SYSVENT divergem pouco e de forma uniforme em relação ao programado, atestando boa capacidade de monitorização. No caso das medições efetuadas pelo Bio-Tek, a divergência em relação ao programado parece ser maior, de sinal contrário ao observado com o SYSVENT, e com incremento ao longo dos patamares de $\mathrm{V}_{\mathrm{T}}$ programado. Esta situação ocorre de forma independente da $\mathrm{C}$, numa magnitude que não parece ter impacto clínico - tanto mais que corresponde, no máximo, a uma média de diferenças de $-8,7 \%$. Este padrão, associado às medições do Bio-Tek, é menos notório na PEEP e não ocorre na $\mathrm{P}_{\text {insp }}$ (vide infra).

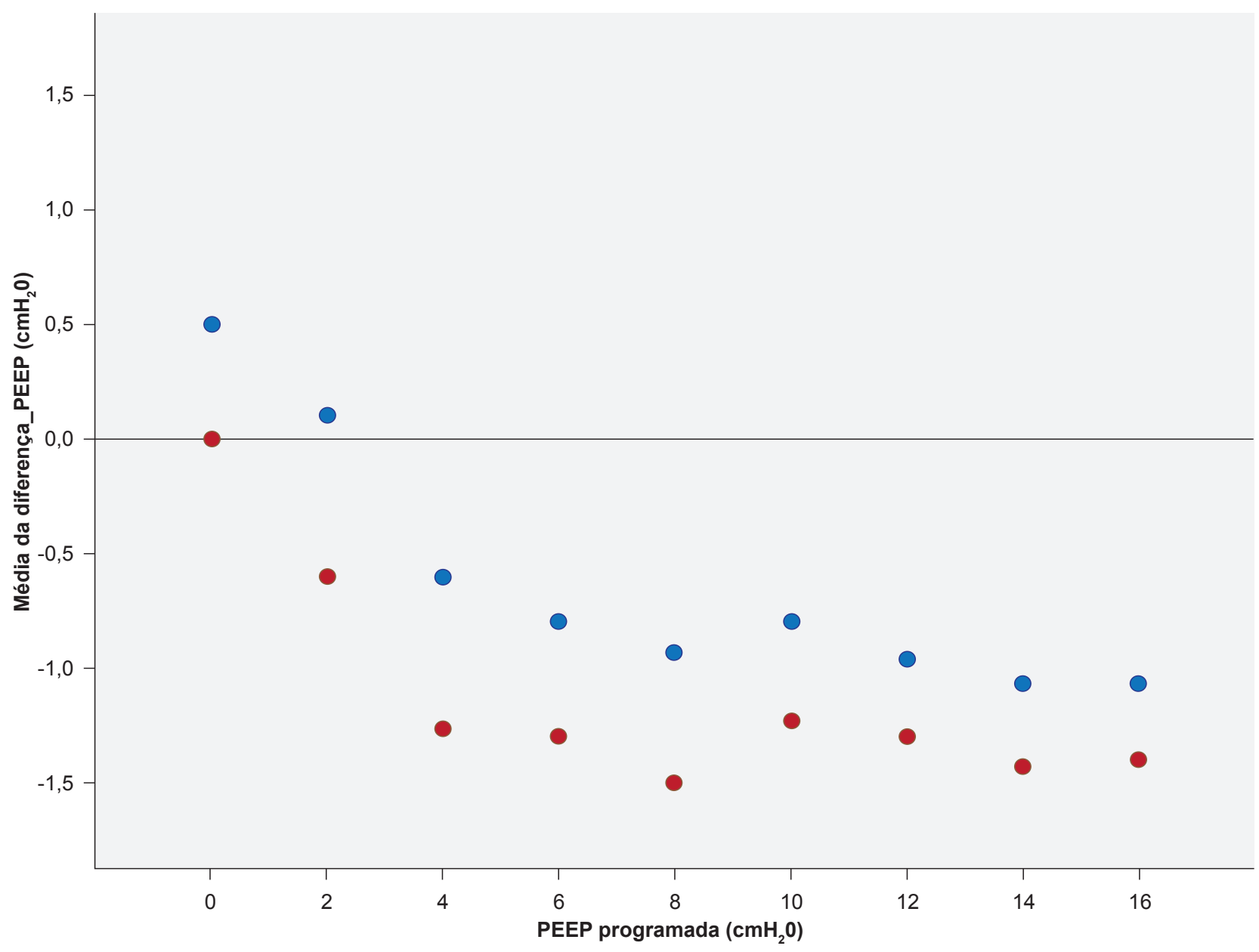

SYSVENT

Bio-TEK

Figura 4 - Distribuição das médias das diferenças, expressas em $\mathrm{cmH}_{2} \mathrm{O}$, entre PEEP medida no SYSVENT e no Bio-Tek e PEEP programada 
A literatura não esclarece a amplitude expectável das diferenças encontradas entre $P_{\text {insp }}$ programada e $P_{\text {insp }}$ medida. Todavia, quando, no âmbito do presente estudo, se compararam as pressões, estas diferenças foram inferiores ou iguais a $3,9 \mathrm{cmH}_{2} \mathrm{O}$, facto que, como já referido, constitui uma irrelevância clínica. Independentemente do desvio em relação aos valores programados, verificou-se que as diferenças encontradas pelos dois equipamentos se sobrepõem. Isto significa que o ventilador tem uma boa capacidade de monitorização, ou seja, que os valores apresentados no ecrã do ventilador correspondem àqueles que estão efetivamente a ser entregues.

$\mathrm{O}$ comportamento do ventilador em relação à PEEP mostrou, igualmente, uma variação entre o medido e o programado clinicamente aceitável. Genericamente, os valores medidos de PEEP são inferiores ao programado, sobretudo pelo Bio-Tek, e esta diferença parece agravar-se com o incremento da PEEP programada. Todavia, a magnitude do desvio entre o programado e o medido não antecipa nenhum impacto clínico.

Estes resultados são indicativos de um bom comportamento mecânico do ventilador, e de uma boa monitorização, com uma variabilidade dentro de um espectro que, sob o ponto de vista clínico, é aceitável. A diferença de medição entre o SYSVENT e o Bio-Tek, cuja causa pode radicar em diferenças intrínsecas na performance de medição de qualquer um dos equipamentos, não ultrapassa os limites de aceitabilidade clínica. Não obstante, a possibilidade de utilizar novos equipamentos de simulação e de medição em testes ulteriores (já previstos), permitirão compreender meIhor a causa das diferenças encontradas.

A metodologia utilizada nestes testes de bancada tentou simular a prática clínica habitual, facto que nos parece representar um conceito metodológico relevante. Indexámos a parametrização do ventilador (fosse em VC ou PC) a uma gama de $V_{T}$ que inclui os volumes mais frequentemente utilizados, tendo por referência um peso corporal de $70 \mathrm{~kg}$ e um espectro terapêutico de $\mathrm{V}_{\mathrm{T}}$ dentro de uma estratégia protetora de pulmão $(6-8 \mathrm{~mL} / \mathrm{kg})$. Fizemos variar as condições da mecânica ventilatória apenas na dimensão da compliance, com o objetivo de introduzir o menor número de variáveis na análise dos resultados. Contrariamente, estudos similares optam por fazer variar as condições da mecânica ventilatória (compliance e resistência), tentando simular contextos clínicos de normalidade, de baixa compliance (ARDS) e de alta resistência (doenças obstrutivas). ${ }^{4,8}$ Reconhecemos que a vantagem de manter uma homeostasia durante as medições, fazendo variar apenas a compliance, limita a amplitude de testagem do ventilador, facto que pode vir a ser colmatado em estudos de bancada futuros.

Este estudo tem três limitações:

1) Relacionado com os equipamentos: foram já descritas as limitações técnicas do pulmão artificial. Esta limitação restringe a sua latitude de experimentação, mas não põe em causa os resultados, nem o conceito que pretendemos provar. Estão previstos ensaios de bancada adicionais com outro tipo de equipamento de teste, que permita ultrapassar esta dificuldade.

2) Número de leituras: a literatura não é consensual sobre a forma de amostragem das leituras. Alguns estudos usam sistemas automatizados que permitem a gravação da medição em causa ${ }^{2-4}$; outros, como o nosso, fazem leituras manuais (três a cinco leituras). ${ }^{1}$ É expectável que um número maior de leituras para cada patamar da variável de interesse aumente a robustez dos resultados. Este ajuste metodológico está previsto com os novos testes de bancada, para os quais será disponibilizado um equipamento de teste e medição com capacidade para gravação das medições.

3) Parâmetros analisados: para além de $V_{T e}$ e de $P_{\text {insp }}$, há um conjunto mais alargado de parâmetros que, no contexto do desenvolvimento de um ventilador, devem ser avaliados. Destes, o fluxo é provavelmente o mais relevante. A simulação em cenários nos quais se possam fazer variar não só $\mathrm{C}$, mas também $\mathrm{R}$ e a fuga, são limitações já enunciadas e justificadas. No presente estudo, cujo principal objetivo era provar o conceito do ventilador SYSVENT OM1, os autores optaram por restringir a análise a parâmetros fundamentais da ventilação (volume e pressão), facto que constitui uma limitação. É, todavia, unanimemente reconhecido por todos os autores que, no processo iterativo a que este ventilador está sujeito, os novos testes de bancada, já com recurso a equipamentos de medição com maior capacidade e funcionalidades, deverão contemplar um painel de avaliação mais amplo.

\section{CONCLUSÃO}

Este estudo prova o conceito de que o SYSVENT OM1 tem uma performance de funcionamento, para os parâmetros testados que está de acordo com as premissas inicialmente definidas. Posto isto, os autores consideram estarem reunidas as condições para estudos subsequentes com vista à utilização clínica do SYSVENT OM1, nos quais se incluem novos testes de bancada sempre que necessário ou exigido. Assim, estão planeados novos testes de bancada, testes in vivo (em laboratório veterinário) e testes de segurança elétrica e eletromagnética, que antecedem a submissão deste projeto à comissão científica e à comissão de ética, com a intenção de avaliar o funcionamento do SYSVENT em ambiente controlado de cuidados intensivos em adultos.

\section{PROTEÇÃO DE PESSOAS E ANIMAIS}

Os autores declaram que os procedimentos seguidos estavam de acordo com os regulamentos estabelecidos pelos responsáveis da Comissão de Investigação Clínica e Ética e de acordo com a Declaração de Helsínquia da Associação Médica Mundial actualizada em 2013.

\section{CONFIDENCIALIDADE DOS DADOS}

Os autores declaram ter seguido os protocolos do seu centro de trabalho acerca da publicação de dados. 


\section{CONFLITOS DE INTERESSE}

Os autores declaram não ter conflitos de interesses relacionados com o presente trabalho.

\section{REFERÊNCIAS}

1. Medicines \& Healthcare Products Regulatory Agency. Rapidly manufactured ventilator system (RMVS). [consultado $2020 \mathrm{abr}$ 6]. Disponivel em: https://www.gov.uk/government/publications/ specification-for-ventilators-to-be-used-in-uk-hospitals-during-thecoronavirus-covid-19-outbreak/rapidly-manufactured-ventilator-systemrmvs.

2. Chatburn RL, El-Khatib M, Mireles-Cabodevila E. A taxonomy for mechanical ventilation: 10 fundamental maxims. Respir Care. 2014;59:1747-63.

3. Burrows B, Huang N, Hughes R, Johnston R, Kilburn K, Kuhn C, et al. Pulmonary terms and symbols. A report of the ACCP-STS Joint Committee on Pulmonary Nomenclature. Chest. 1975;67:583-93.

4. Aissam Lyazidi A, Thille A, Carteaux G, Galia F, Brochard L, Richard

\section{FONTES DE FINANCIAMENTO}

Este trabalho não recebeu qualquer tipo de suporte financeiro de nenhuma entidade no domínio público ou privado.

JC. Bench testing of pressure support ventilation with three different generations of ventilators. Intensive Care Med. 2010;36:2074-80.

5. Wallon G, Bonnet A, Guérin C. Delivery of tidal volume from four anaesthesia ventilators during volume-controlled ventilation: a bench study. Br J Anaesth. 2013;110:1045-51.

6. L'Her E, Roy A. Bench tests of simple, handy ventilators for pandemics: performance, autonomy, and ergonomy. Respir Care Med. 2011;56:75160

7. L'Her E, Roy A, Marjanovic N. Bench-test comparison of 26 emergency and transport ventilators. Critical Care. 2014;18:506-20.

8. Zanetta G, Robert D, Guérin C. Evaluation of ventilators used during transport of ICU patients - a bench study. Intensive Care Med. 2002;28:443-51. 